\title{
¿Qué Determina el Desempeño en la Toma de Decisiones de Hombres y Mujeres?
}

\section{What Determines job Performance in Decision Making by Men and Women?}

\author{
Alicia Arenas Moreno \\ Universidad de Sevilla
}

\author{
Carmen Tabernero Urbieta \\ Universidad de Córdoba
}

\author{
Elena Briones Pérez \\ Universidad de Valladolid
}

\begin{abstract}
Resumen. El presente trabajo pretende analizar el efecto del sexo sobre el desempeño en una tarea compleja de toma de decisiones. El rendimiento en tareas de logro está ligado a las dinámicas autorreguladoras, como la autoeficacia, las metas personales y el compromiso con la tarea, determinadas por variables disposicionales como la orientación de las metas o, estudiada más recientemente, la orientación hacia los errores. Concretamente, tratamos de comparar los procesos de autorregulación y factores disposicionales entre chicos y chicas estudiantes universitarios ante la realización de una tarea que simula la implementación de la innovación y el cambio en una organización. Los resultados muestran que son las mujeres quienes manifiestan una clara tendencia a comunicar los errores y se muestran menos preocupadas por demostrar competencia ante los demás. Sin embargo, ante las dificultades mostrarían sentirse menos capaces de afrontarlas, más angustiadas y se plantearían metas más bajas para su desempeño, lo que les llevaría finalmente a alcanzar un rendimiento inferior.

Palabras clave: sexo, toma de decisiones, orientación de las metas, orientación hacia los errores, procesos de autorregulación, desempeño.
\end{abstract}

\begin{abstract}
This study attempts to analyse the effect of gender on performance in a complex decisionmaking task. Performance in achievement tasks is linked to self-regulatory processes such as self-efficacy, self-set goals, and task commitment, determined by dispositional factors such as goal orientation or, as recently discovered, error orientation. Specifically, we try to compare self-regulation processes and dispositional factors of male and female university students in a complex task that simulates innovation and change implementation in a fictitious organization. Results show that women display a clear tendency to communicate errors and they are less worried about proving their own competence in front of others. Women, however, feel less able to cope with difficulties, are more distressed, and choose easier goals for their performance, which leads them ultimately to achieve worse performance.

Keywords: gender, decision making, goal orientation, error orientation, self-regulatory-processes, performance.
\end{abstract}

Las particularidades de la sociedad actual exigen a sus individuos la adquisición y el desarrollo de proyectos de vida caracterizados por actitudes basadas en la percepción de capacidad, en el conocimiento de sus metas, intereses y habilidades y en la utilización de estrategias de decisión basadas en la planificación. En esta sociedad, el desarrollo de una integración laboral satisfactoria es un derecho de todos, cuyo pilar fundamental es el respeto a la diversidad. Así, los procesos que intervienen en la identidad profesional están profundamente marcados por la variable sexo (Sarrió, Barberá, Ramos y Candela, 2002).

Desde los primeros años de edad, niños y niñas aprenden e incorporan creencias diferenciales asociadas con los sexos, en las que se considera que los niños

Este artículo se ha realizado en parte gracias al proyecto de investigación financiado por el Ministerio de Ciencia y Tecnología PSI200907423. La correspondencia sobre este artículo deberá ser dirigida a Alicia Arenas Moreno, Universidad de Sevilla, Facultad de Psicología, C/ Camilo J. Cela, s/n, 41018 Sevilla, e-mail: aarenas@us.es tienden a ser competitivos, aventureros, disfrutan de tareas que implican riesgos, a la vez que están más interesados por tareas que están relacionadas con las matemáticas, las ciencias y la tecnología. A su vez, se considera a las niñas cooperativas, cariñosas, caritativas, intuitivas, más emotivas, menos racionales y más interesadas por tareas relacionadas con la lectura, la escritura, las artes y las relaciones interpersonales. Estas creencias estereotipadas acerca de los sexos se desarrollan a edades muy tempranas (Burke, 1996), aumentan en complejidad en la niñez y la adolescencia y se vuelven más pronunciadas con la edad (Valiante, 2000). Este conocimiento es integrado por cada persona e impregna todos los órdenes de la vida.

En este sentido, el meta-análisis llevado a cabo por Byrnes, Miller y Schafer (1999) apoya la idea de que los hombres asumen riesgos con mayor probabilidad que las mujeres. Pero también las diferencias de sexo varían de acuerdo al contexto y al nivel de edad. Así, los hombres tenderían a encontrarse con fracasos $\mathrm{u}$ otras consecuencias negativas más a menudo que las 
mujeres, mientras que las mujeres tenderían a experimentar éxito menos a menudo de lo que deberían. En este sentido, algunos autores (p. ej., Weller, Levin y Bechara, 2009; Weller, Levin y Denburg, 2011) destacan que los hombres superan en rendimiento a las mujeres en tareas de toma decisiones de riesgo pero que esta tendencia se vería modificada con la edad.

Sin embargo, si consideramos las conductas de riesgo en un sentido más amplio, Byrnes (1998) sugiere que existe un incremento de la adopción de riesgos con la edad porque es más probable que los niños se interesen más por ambientes nuevos y no controlados a medida que crecen. Resistir la tentación de arriesgarse requiere de un cierto grado de autorregulación, es decir, sentido de incertidumbre, estrategias correctivas para manejar las distracciones y una tendencia a aprender de los errores. Las diferencias en cuanto al sexo podrían estar reflejando un doble estándar con respecto al control de los padres (serían más restrictivos con las chicas), sobreconfianza en los chicos y menos conocimiento de las estrategias de auto-corrección en los chicos.

Algunos autores (p. ej., Irwin y Millstein, 1991) han explicado este incremento por cambios periódicos en la maduración biológica, la capacidad cognitiva (perspectiva de futuro), las autopercepciones (autoestima, por ejemplo), las percepciones del ambiente social (p. ej., influencias de los padres e iguales), los valores personales (p. ej., independencia), la percepción del riesgo (disposición optimista) y las características del grupo de iguales (p. ej., edad de los iguales).

No queda claro si estos hallazgos reflejan el hecho de que los hombres se enfrentan a contextos que inducen al riesgo más a menudo que las mujeres o si las mujeres tienen una mayor capacidad para negociar consigo mismas y autorregularse a través de este tipo de situaciones. Otra explicación puede estar en una diferencia en competitividad vinculada al sexo, lo que estaría teniendo importantes implicaciones laborales que comentaremos más adelante. Además, la elección arriesgada es más valorada culturalmente, lo que en la literatura sobre la percepción del riesgo se ha denominado tradicionalmente "hipótesis del riesgo como valor" (Kelling, Zirkes y Myerowitz, 1976).

Por otra parte, existe un amplio cuerpo de investigación que analiza los patrones motivacionales que despliegan ambos géneros en situaciones de logro (p. ej., Middleton y Midgley, 1997; Pajares y Schunk, 2001). Dweck $(1989,1999)$ recogió estudios en los que mostraba que las niñas alcanzaban el mismo nivel de desempeño que los niños en matemáticas durante los primeros años escolares (e incluso les sobrepasaban en tareas de carácter verbal). Durante los últimos años de colegio y en la educación media, sin embargo, los chicos aventajaban a las chicas y se mantenían a la cabeza en matemáticas.

Específicamente, el hecho de que chicos y chicas desplieguen a menudo diferentes patrones motivacio- nales y que las áreas académicas en cuestión -matemáticas y comprensión verbal- difieran bastante, aparte de las habilidades que requieren, sugiere que quizá los patrones motivacionales estén contribuyendo a estas discrepancias de logro. En general, las chicas despliegan expectativas menos seguras, atribuyen con mayor frecuencia el fracaso a una falta de capacidad, muestran menor preferencia por tareas nuevas y desafiantes y afrontan peor las dificultades o la confusión (p. ej., Dweck, 1999).

El estudio que presentamos trata de explorar el papel que juega la variable sexo en el desempeño en tareas complejas en su interacción con algunas variables disposicionales como la orientación de las metas, directamente implicada en el desempeño en tareas de logro, y la orientación hacia los errores. Además, consideramos que la variable sexo influirá en los mecanismos autorreguladores que se ponen en práctica para poder explicar de forma más adecuada el desempeño en tareas complejas (juicios de eficacia para la tarea, metas, reacciones afectivas y compromiso con la tarea).

La orientación de las metas se refiere a un conjunto de metas de orden superior que ayudan a determinar las actitudes y conductas en las situaciones de logro (p. ej., Dweck y Leggett, 1988) y el trabajo empírico ha conectado estas metas con variables de resultado importantes tales como el desempeño, la búsqueda de feedback o la satisfacción (p. ej., Elliot, McGregor y Gable, 1999; VandeWalle y Cummings, 1997). Individuos con un nivel más alto en la orientación de sus metas hacia el aprendizaje o dominio guiarán su comportamiento por el deseo de desarrollar su competencia en la tarea que están realizando, es decir, por el deseo de aprender cómo mejorar sus capacidades. Simultáneamente, esas mismas personas pueden diferir en los niveles de su orientación de las metas hacia la ejecución o el desempeño, en las que desean demostrar su competencia o evitar demostrar su incompetencia ante los demás en esa tarea.

Por otra parte, los reguladores clave de la conducta intencional incluyen autoeficacia o autoevaluación de la capacidad para realizar la tarea, evaluación del desempeño frente a una meta o estándar y las reacciones afectivas, que incluyen la satisfacción.

La autoeficacia no sólo garantiza un nivel de esfuerzo e implicación en el desempeño en tareas complejas, también es la motivación necesaria para persistir ante los fracasos, para afrontar los riesgos y posibles errores en el proceso de toma de decisiones. Aquellas personas que sostienen un nivel elevado de autoeficacia desarrollan con mayor facilidad espirales ascendentes en este proceso (Lindsey, Brass y Thomas, 1995). Estas espirales se construyen desde la propia percepción de capacidad para afrontar las dificultades y en ellas la autoeficacia determina el nivel de esfuerzo y el interés en lograr un nivel de metas elevado para lograr el mejor desempeño. Sin embargo, cuando la persona 
no se siente capaz para afrontar la tarea se desarrollan espirales descendentes como resultado de interpretar el feedback de resultado. Al realizar un juicio de atribución poco adaptativo la persona se culpabiliza de sus resultados, abandona cualquier esfuerzo y finalmente entra en un estado de malestar que le lleva a abandonar la tarea. En este sentido, algunos autores (p. ej., Virtanen y Nevgi, 2010) han encontrado cómo hombres y mujeres difieren en su autorregulación frente a tareas de aprendizaje y en la aplicación de sus estrategias motivacionales a la tarea en determinadas carreras profesionales.

Algunas investigaciones han demostrado que las mujeres entran con mayor facilidad en estas espirales descendentes y se culpabilizan a sí mismas de su bajo desempeño en situaciones de logro donde están presentes aspectos nuevos y difíciles. Desde la teoría social cognitiva (Bandura, 1986, 1997, 1999), creemos que las diferencias de género en los juicios de autoeficacia para el desempeño en tareas laborales podrían ser entendidas como la clave que explicaría esas barreras cognitivas que impiden a las mujeres alcanzar un mejor desempeño. Aún hoy en día nos encontramos con creencias generalizadas de que hay trabajos o habilidades claramente masculinos o femeninos.

A la hora de interpretar las diferencias de sexo en los juicios de autoeficacia debemos considerar que ambos grupos responden a los instrumentos de autoinforme desde un baremo muy diferente. Así, mientras las mujeres tienden a ser más modestas al informar sobre sus niveles de capacidad, los hombres tienden a sentirse orgullosos de sus resultados. En otras palabras, es muy probable que los chicos muestren unos niveles elevados de confianza en capacidades que pueden no tener, al tiempo que tienden a mostrar sobreconfianza en aquellas habilidades en las que son competentes.

Por supuesto, esas barreras sociales que generan estereotipos en relación al sexo al construir trabajos masculinos o femeninos también influyen en los juicios de autoeficacia. Así, los estereotipos de rol de género son parcialmente responsables de las diferencias en la elección de carreras, del desarrollo profesional, de los juicios de confianza y la valoración percibida para realizar determinadas carreras, tareas o actividades laborales o sociales (Hackett y Betz, 1991). Alta autoeficacia se asocia con metas personales más altas, compromiso más fuerte con esas metas, mejor resolución de problemas y estrategias analíticas, más esfuerzo y persistencia ante las dificultades y una tendencia a interpretar el pobre desempeño de forma constructiva más que debilitadora (Bandura, 1997; Debowski, Wood y Bandura, 2001; Tabernero y Wood, 1999; Wood, Kakebeeke, Debowski y Frese, 2000).

Basándonos en la investigación revisada, proponemos que las disposiciones personales relacionadas con la motivación para el aprendizaje de tareas complejas, como la orientación hacia los errores, la orientación de las metas y los juicios de autoeficacia previos, están relacionados con la variable sexo, así como con los mecanismos autorreguladores y el desempeño en una tarea compleja de toma de decisiones. Sobre la base de estos argumentos avanzamos las siguientes hipótesis:

H1: Los participantes varones presentarán una orientación hacia los errores y una orientación de sus metas más adaptativa para tomar decisiones complejas (orientación hacia el aprendizaje) que las mujeres participantes en el estudio.

H2: Los hombres presentarán una mayor confianza en su capacidad para solventar las dificultades que conlleva una tarea compleja de toma de decisiones que las mujeres. Igualmente, esperamos que los varones muestren unas metas más elevadas, un compromiso con la tarea y un estado afectivo más positivo que sus compañeras en la toma de decisiones.

H3: El género, la orientación hacia el aprendizaje y los mecanismos reguladores determinarán el desempeño final logrado.

\section{Método}

\section{Muestra}

La muestra está compuesta por un total de 104 estudiantes de distintos cursos de la licenciatura de Psicología de una universidad del centro de España; el $46,2 \%$ pertenece al primer curso y más del $80 \%$ son mujeres. El 96,2\% de los participantes indica ser menor de 26 años.

\section{Tarea y procedimiento}

El estudio fue presentado como unas sesiones de dos horas de entrenamiento y aprendizaje en toma de decisiones a través de un simulador. Su participación fue recompensada económicamente. En primer lugar, los participantes contestaron, garantizando su anonimato, a un cuestionario en el que se incluían medidas de algunos de los factores que pueden estar determinando el desempeño ante una tarea compleja de toma de decisiones.

Una vez que todos hubieron completado el cuadernillo, se les explicaba en qué consistía la tarea que debían realizar. Consistía en un programa de simulación, concebido como herramienta para la formación en toma de decisiones, en el que los participantes debían introducir un elemento innovador en la organización simulada tratando de lograr el mayor número de adoptadores posible (Angehrn, 2004). En el programa de simulación se presenta el reto de persuadir a los trabajadores de una filial de una gran compañía para que adopten un cambio en el sistema de gestión de la información. El 
organigrama de la filial presenta 22 directores de diferentes departamentos y dos asesores de la dirección. Para la mayoría de los trabajadores la adopción de cambios supone un esfuerzo extra por lo que éstos suelen ser rechazados. El programa presenta numerosas iniciativas que el individuo puede utilizar para persuadir a los trabajadores para que acepten la incorporación de cambios en su trabajo. El programa de simulación permite aprender que las iniciativas de cambio deben adoptarse siguiendo un patrón secuencial: dar a conocer el nuevo sistema propuesto, generar interés en las personas hacia el cambio, dar la posibilidad de ponerlo a prueba y finalmente adoptar el sistema de cambio. Así, hay iniciativas para dar a conocer el nuevo sistema de gestión (p. ej., publicar información del nuevo sistema de gestión en la intranet de la organización), para generar interés (p. ej., implementar un programa formación de directivos) o para poner a prueba el cambio propuesto (p. ej., organizar un taller). El reto de persuadir al máximo número de adoptantes debe lograrse en 120 días, sabiendo que cada iniciativa que se desarrolla conlleva un número de días (p. ej., pedir consejo a un directivo supone dos días de trabajo). El software se les explicó a través de una DEMO, presentada en versión powerpoint. A continuación, respondieron a una serie de preguntas relacionadas con su percepción de capacidad para alcanzar determinados resultados en la tarea, sus metas con respecto a la misma, compromiso y estado afectivo para afrontarla. Por último, antes de abandonar la sesión con el simulador se les pidió que respondieran de nuevo a las cuestiones que hacían referencia a los mecanismos autorreguladores.

\section{Medidas}

\section{Variables disposicionales}

Orientación hacia los Errores. Utilizamos una versión reducida del cuestionario Error Orientation Questionnaire (EOQ), que hace referencia a las actitudes y el afrontamiento de los errores en el lugar de trabajo. Se pedía a los participantes que señalaran en qué medida estaban de acuerdo con cada uno de los enunciados en una escala tipo Likert de 5 puntos (siendo $1=$ 'no del todo cierto' y 5='totalmente cierto'). Rybowiak, Frese, Garst y Batinic (1999) elaboraron ocho subescalas, en este estudio tomamos 30 de los 37 ítems originales agrupados en seis dimensiones. La dimensión 'aprendizaje de los errores' obtiene una fiabilidad de $\alpha=.87$; la dimensión 'asumir el riesgo de cometer errores' con un $\alpha=.75$; la dimensión 'estrés por cometer errores' alcanza un coeficiente de $\alpha=.82$; la dimensión 'ocultar los errores' tiene una fiabilidad de .79; la dimensión 'comunicación de los errores' alcanza un $\alpha=.73$ y la dimensión 'reflexionar sobre la ocurrencia de errores' con un $\alpha=.81$.

Autoeficacia. Utilizamos una adaptación del micro- análisis que, tradicionalmente, se ha empleado para evaluar los juicios de capacidad en la toma de decisiones (Tabernero y Wood, 1999; Wood y Bandura, 1989). El microanálisis consta de ocho ítems ordenados gradualmente según su nivel de dificultad. Los ítems evalúan el nivel de confianza para afrontar diferentes situaciones académicas de distinto grado de dificultad. Los participantes debían reflejar si se sentían capaces de afrontar cada una de las situaciones planteadas y con qué nivel de confianza (0-10). La escala muestra un coeficiente de fiabilidad de .81 .

Orientación de las Metas. Para evaluar la orientación de las metas en el ámbito organizacional en el que se desarrolla la tarea de simulación se empleó una adaptación del cuestionario que VandeWalle desarrolló en este contexto concreto y que denominó Work Domain Goal Orientation Instrument (VandeWalle, 1997). Los ítems se agrupan en tres dimensiones: una dimensión de aprendizaje (learning goal orientation), que mantendrían aquellas personas que consideran los errores o fallos en el lugar de trabajo como parte del aprendizaje; una dimensión de aproximación a la ejecución (prove performance goal orientation), que caracterizaría a aquellas personas que tratan de realizar su tarea lo mejor posible, centrándose en los resultados; y una dimensión de evitación de la ejecución (avoid performance goal orientation), que les llevaría a evitar juicios negativos por parte de los demás. Cada una de las dimensiones aparece medida a través de cuatro ítems que los participantes deben contestar en una escala tipo Likert de 7 puntos, siendo $1=$ 'totalmente en desacuerdo' y 7='totalmente de acuerdo'. Las tres dimensiones presentan un nivel de fiabilidad adecuado ( $\alpha=.80, \alpha=.90, \alpha=.73$, respectivamente $)$.

\section{Mecanismos autorreguladores (medidos antes y después de la simulación)}

Autoeficacia. Evaluada a través de seis ítems que hacen referencia a la creencia sobre la propia capacidad para alcanzar diferentes resultados en el programa de simulación. Para cada una de las cuestiones debían realizar dos juicios: "¿Me siento capaz de lograr este resultado?", al que deben responder "sî́" o "no" (Nivel de autoeficacia) y, si su respuesta es afirmativa, deben responder "¿En qué medida me siento capaz de lograr este resultado?", en una escala de 1 (ninguna confianza) a 10 (total confianza) puntos (Intensidad de la autoeficacia). Los coeficientes de fiabilidad de esta escala fueron de .93 (antes) y .87 (después).

Metas para la tarea. Medidas a través de dos cuestiones, una que hace referencia al número de adoptadores que se plantean conseguir en el tiempo de la simulación, y otra a cuántos puntos pretenden lograr. La medida que se realiza después de la simulación hace alusión al número de adoptadores y de puntos que se plantearían lograr en el futuro. 
Compromiso. Evaluado a través de los ítems "¿En qué medida te sientes comprometido con la meta seleccionada?” y "¿En qué medida te sentirías comprometido con la meta seleccionada en el futuro?", en una escala de 1 (nada) a 9 (totalmente comprometido).

Reacciones Afectivas. Se evalúan a través de un diferencial semántico (Snider y Osgood, 1969) con una escala de seis puntos, en la que tras la cuestión “CCómo te sientes en este momento?”, el participante debería señalar el valor que más se ajustase a su respuesta para cuatro pares de adjetivos en una escala de uno a seis, siendo las escalas: contento (1) - triste (6), tenso (1) - relajado (6), bien (1) - mal (6) y calmado (1) - ansioso (6); así, puntuaciones más altas indicarían un estado de ánimo más negativo. Los coeficientes de fiabilidad alcanzaron un alfa de .71, para la medida antes de la simulación, y .79 para la medida tras la simulación.

\section{Desempeño en el programa de simulación}

El programa nos permite recoger una serie de datos que pueden ser considerados como variables dependientes del estudio. En el presente trabajo tomamos como indicador del desempeño el número de adoptadores logrados. Este es el objetivo más importante para cada participante del programa, sabemos que hay 24 personas en el organigrama de los puestos directivos de la filial y hay un máximo de 22 adoptadores que podrían lograr con un desempeño perfecto. El programa presenta un panel de control con el listado de los 24 directores generales y el estado en el que se encuentran para llegar a adoptar el programa. Los participantes pueden comprobar a través de una barra de progreso para cada directivo si éste conoce el programa de gestión del cambio, tiene interés por él, lo ha puesto a prueba o ha llegado a adoptarlo. Dado que la meta propuesta para los participantes es lograr el máximo número de adoptadores posible, la variable dependiente considerada fue el número de adoptadores logrados.

\section{Resultados}

\section{Relaciones entre las variables de estudio y el desempeño final en el programa de simulación}

Como se puede ver en la Tabla 1, la variable sexo $(0=$ hombres; $1=$ mujeres $)$ correlaciona negativa y significativamente con la orientación de las metas hacia los resultados. Así, son las mujeres quienes manifiestan una menor orientación hacia el desempeño. La autoeficacia correlaciona en sentido positivo con la orientación de las metas hacia el aprendizaje. La autoeficacia y la orientación de las metas hacia el aprendizaje presentan, además, relaciones significativas similares. Así, ambas correlacionan negativamente con la orientación de las metas de evitación.

En cuanto a los mecanismos autorreguladores medidos antes y después de llevar a cabo la tarea, la Tabla 2 muestra cómo la variable sexo presenta relaciones significativas negativas con la autoeficacia, tanto antes de realizar la simulación como después, las metas que se plantean los participantes en cuanto al número de adoptadores a alcanzar, tanto antes como después de la tarea, con los puntos a lograr y el compromiso antes de la tarea. En todos los casos, son las mujeres quienes menos capaces se sienten para realizar la simulación, tanto antes como después de llevarla a cabo, quienes se proponen metas más bajas y menor compromiso manifiestan con dichas metas.

\section{Efecto del sexo sobre las variables disposicionales evaluadas}

Como comentamos antes, nos planteamos explorar si existen diferencias en cuanto al sexo en los distintos aspectos disposicionales evaluados, para ello llevamos a cabo varios análisis de varianza (ANOVA). En cuanto a la orientación de los participantes hacia los errores, los datos muestran diferencias entre hombres y mujeres únicamente para la dimensión de comunicación de los errores $\left(\mathrm{F}(1,101)=2.78, \mathrm{p}<.10, \eta^{2}=.027\right)$,

Tabla 1. Coeficientes de correlación, medias y desviaciones típicas entre la variable sexo y las variables disposicionales analizadas

\begin{tabular}{|c|c|c|c|c|c|c|}
\hline & \multirow{2}{*}{$\frac{1}{-}$} & \multirow[t]{2}{*}{2} & \multirow[t]{2}{*}{3} & \multirow[t]{2}{*}{4} & \multirow[t]{2}{*}{5} & \multirow[t]{2}{*}{6} \\
\hline 1. Sexo & & & & & & \\
\hline 2. Comunicar & .16 & - & & & & \\
\hline & -.17 & .15 & - & & & \\
\hline $\begin{array}{l}\text { 3. Autoeficacia } \\
\text { 4. O. Metas Aprendizaie }\end{array}$ & -.15 & .17 & $.60^{* *}$ & - & & \\
\hline \multirow{2}{*}{$\begin{array}{l}\text { 5. O. Metas Resultados } \\
\text { 6. O. Metas Evitación }\end{array}$} & $-.20^{*}$ & -.02 & .14 & .10 & - & \\
\hline & .06 & -.16 & $-.48 * *$ & $-.38^{* *}$ & .11 & - \\
\hline \multicolumn{2}{|l|}{ Media } & 4.03 & 5.33 & 5.36 & 4.40 & 3.30 \\
\hline \multicolumn{2}{|l|}{ D.T. } & .60 & 1.71 & .88 & 1.40 & 1.12 \\
\hline \multicolumn{7}{|l|}{$\begin{array}{l}* p<.05 \\
* * * 0.01\end{array}$} \\
\hline
\end{tabular}


Tabla 2. Coeficientes de correlación, medias y desviaciones típicas entre la variable sexo y los mecanismos autorreguladores evaluados antes y después de realizar la tarea

\begin{tabular}{|c|c|c|c|c|c|c|c|c|c|c|c|c|}
\hline Variables & 1 & 2 & 3 & 4 & 5 & 6 & 7 & 8 & 9 & 10 & 11 & 12 \\
\hline 1. Sexo & - & & & & & & & & & & & \\
\hline 2. Autoeficacia 1 & $-.23^{*}$ & - & & & & & & & & & & \\
\hline 3. Autoeficacia 2 & $-.34 * *$ & $.41 * *$ & - & & & & & & & & & \\
\hline 4. Reacciones afectivas 1 & .16 & -.09 & -.05 & - & & & & & & & & \\
\hline 5. Reacciones afectivas 2 & .02 & .06 & $-.36^{* *}$ & $.28^{* *}$ & - & & & & & & & \\
\hline 6. Metas adoptadores 1 & $-.19^{*}$ & $.61 * *$ & .12 & -.10 & .04 & - & & & & & & \\
\hline 7. Metas puntos 1 & $-.27 * *$ & $.57^{* *}$ & $.21^{*}$ & -.08 & -.07 & $.81^{* *}$ & - & & & & & \\
\hline 8. Compromiso 1 & $-.21^{*}$ & $.25^{*}$ & $.21^{*}$ & $-.24 *$ & -.10 & $.30^{* *}$ & $.39 * *$ & - & & & & \\
\hline 9. Metas adoptadores 2 & $-.23^{*}$ & $.31 * *$ & $.65^{* *}$ & -.09 & $-.42 * *$ & $.28^{* *}$ & $.34 * *$ & $.25^{*}$ & - & & & \\
\hline 10. Metas puntos 2 & -.16 & $.25^{*}$ & $.50 * *$ & -.07 & $-.39 * *$ & .19 & $.28 * *$ & .15 & $.72 * *$ & - & & \\
\hline 11. Compromiso 2 & -.17 & .18 & $.28 * *$ & -.01 & $-.21 *$ & $.20 *$ & .20 & $.51 * *$ & $.29 * *$ & $.21 *$ & - & \\
\hline 12. Adoptadores & -.16 & .02 & $.40^{* *}$ & -.06 & $-.41 * *$ & -.01 & .03 & .12 & $.41 * *$ & $.52 * *$ & .18 & - \\
\hline Media & & 4.51 & 4.19 & 2.94 & 2.46 & 13.08 & 145.85 & 6.87 & 11.51 & 147.47 & 7.35 & 5.98 \\
\hline D.T. & & 2.24 & 2.30 & .081 & .97 & 5.31 & 54.02 & 1.30 & 4.76 & 48.45 & 1.28 & 3.82 \\
\hline
\end{tabular}

aunque éstas son marginales. Así, son las mujeres quienes manifiestan una actitud más positiva hacia los mismos y la posibilidad de comunicárselos a los demás $\left(\mathrm{M}_{\text {MUJERES }}=4.07\right.$, d.t.=.58) frente al grupo de hombres $\left(\mathrm{M}_{\mathrm{HOMBRES}}=3.80\right.$, d.t. $\left.=.71\right)$.

Con respecto a los juicios de capacidad para afrontar dificultades en el contexto académico, de nuevo las diferencias entre los dos grupos son marginales $\left(\mathrm{F}(1,99)=3.05, \mathrm{p}<.10, \eta^{2}=.030\right)$, siendo, en este caso, los hombres quienes manifiestan sentirse más capaces a la hora de llevar a cabo tareas complejas en su trabajo cotidiano $\left(\mathrm{M}_{\text {HOMBRES }}=6.03\right.$, d.t. $\left.=1.32\right)$ frente al grupo de mujeres $\left(\mathrm{M}_{\mathrm{MUJERES}}=5.20\right.$, d.t. $\left.=1.76\right)$.

En el caso de la orientación de las metas, aparecen diferencias significativas entre ambos grupos en el caso de la orientación hacia probar la capacidad o mostrar competencia ante los demás $(\mathrm{F}(1,101)=4.06$, $\left.\mathrm{p}<.05, \eta^{2}=.039\right)$. Así, son los hombres quienes muestran una tendencia más clara hacia los resultados $\left(\mathrm{M}_{\text {HOMBRES }}=5.05\right.$, d.t. $\left.=1.02\right)$ que el grupo de mujeres $\left(\mathrm{M}_{\text {MUJERES }}=4.29\right.$, d.t. $\left.=1.44\right)$.

\section{Efecto del sexo sobre los mecanismos autorreguladores}

Cambios en la percepción de capacidad para la tarea. El modelo de medidas repetidas para la percepción de capacidad medida antes y después de la simulación presenta diferencias significativas en función del género $\left(F(1,96)=12.11, p<.001, \eta^{2}=.112\right)$. Sin embargo, las pruebas t para muestras relacionadas para cada grupo no muestran cambios significativos para el grupo de los hombres $(\mathrm{t}(14)=-.48$, n.s.), a pesar de que aumenta ligeramente su nivel de autoeficacia. Por su parte, los cambios para el grupo de mujeres resultan marginales $(\mathrm{t}(82)=1.65, \mathrm{p}<.10)$, en la dirección opues- ta, en este caso informan de un menor nivel de autoeficacia para la tarea tras realizar la simulación. Las pruebas $\mathrm{t}$ para muestras independientes revelan, no obstante, diferencias significativas entre ambos grupos en el caso de la autoeficacia medida antes de realizar la tarea $\left(\mathrm{t}(99)=2.35, \mathrm{p}<.05 ; \mathrm{M}_{\text {HомвRES }}=5.73\right.$, d.t. $=2.39$; $\mathrm{M}_{\text {MUJERES }}=4.29$, d.t. $\left.=2.16\right)$ y para la autoeficacia tras la tarea $\left(\mathrm{t}(96)=3.49, \mathrm{p}<.01 ; \mathrm{M}_{\text {HOMBRES }}=6.00\right.$, d.t. $=2.71$; $\mathrm{M}_{\text {MUJERES }}=3.86$, d.t. $=2.08$ ), siendo en ambos casos el grupo de los hombres quienes informan de mayor percepción de eficacia para la tarea (véase Figura 1).

Metas (número de adoptadores). El modelo de medidas repetidas para el factor sexo sobre las metas evaluadas antes y después de la simulación de nuevo resulta significativo $\left(\mathrm{F}(1,97)=8.29, \mathrm{p}<.01, \chi^{2}=.079\right)$. Las pruebas t para muestras relacionadas muestran que los cambios entre la medida antes y tras la simulación son significativos para el grupo de mujeres $\left(\mathrm{t}(83)=2.26, \quad \mathrm{p}<.05 ; \quad \mathrm{M}_{\text {ANTES }}=12.63, \quad\right.$ d.t. $=5.35$; $\mathrm{M}_{\mathrm{DESPUÉS}}=11.10$, d.t.=4,53), disminuyendo el número de adoptadores que se plantean lograr si volvieran a realizar la simulación. En el caso de los varones, las diferencias no resultan significativas, quizá por el hecho de tratarse de un grupo muy pequeño. Las pruebas t para muestras independientes revelan que existen diferencias significativas entre las metas que se propone el grupo de hombres y el grupo de mujeres tanto si se evalúan antes de la simulación $(\mathrm{t}(101)=1.98, \mathrm{p}<.05$; $\mathrm{M}_{\text {HOMBRES }}=15.50$, d.t. $=4.46 ; \mathrm{M}_{\text {MUJERES }}=12.67$, d.t.=5.38), como tras la misma $\left(\mathrm{t}(97)=2.38, \mathrm{p}<.05 ; \mathrm{M}_{\mathrm{HOM}}\right.$ ${ }_{\text {BRES }}=14.20$, d.t. $=5.29 ; \mathrm{M}_{\text {MUJERES }}=11.10$, d.t. $=4.53$ ), siendo, en ambos casos, los hombres quienes creen poder conseguir mayor número de adoptadores.

Metas (número de puntos). El modelo de medidas repetidas para el factor sexo sobre las metas con respecto al número de puntos evaluadas antes y después de la simulación resulta significativo $(\mathrm{F}(1,96)=7.84$, 
Figura 1. Cambios en los juicios de capacidad para la tarea en función del sexo

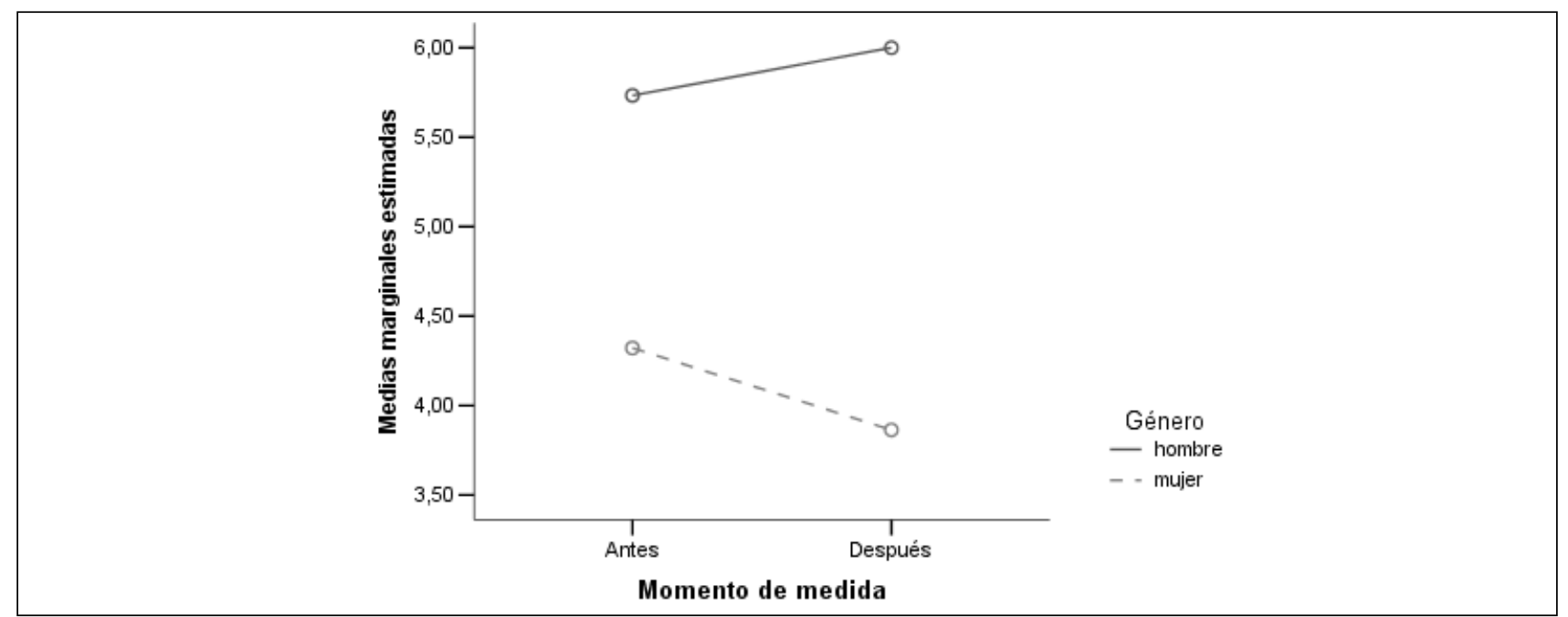

$\left.\mathrm{p}<.01, \eta^{2}=.076\right)$ también en este caso. Sin embargo, las pruebas t para muestras relacionadas no muestran cambios entre la medida antes y tras la simulación ni para el grupo de mujeres ni para el de hombres. Las pruebas $\mathrm{t}$ para muestras independientes revelan que existen diferencias significativas entre las metas que se propone el grupo de hombres y el grupo de mujeres antes de la simulación $\left(\mathrm{t}(99)=2.79, \mathrm{p}<.01 ; \mathrm{M}_{\mathrm{HOMBRES}}=179.31\right.$, d.t.=39.41; $\mathrm{M}_{\text {MUJERES }}=139.55$, d.t.=54.26), pero no tras la misma, siendo los hombres quienes creen poder conseguir más puntos.

Compromiso. El modelo de medidas repetidas para el factor sexo sobre el compromiso evaluado antes y después de la simulación resulta significativo $\left(\mathrm{F}(1,95)=4.73, \mathrm{p}<.05, \eta^{2}=.047\right)$. Las pruebas $\mathrm{t}$ para muestras relacionadas muestran que los cambios entre la medida antes y tras la simulación son significativos para el grupo de mujeres $\left(\mathrm{t}(81)=-4.17, \mathrm{p}<.001 ; \mathrm{M}_{\mathrm{AN}}\right.$ TES $=6.71$, d.t. $=1.22 ; \mathrm{M}_{\mathrm{DESPUÉS}}=7.27$, d.t.=1.33), aumentando el nivel de compromiso si volvieran a realizar la simulación. En el caso de los varones, las diferencias no resultan significativas. Las pruebas t para muestras independientes revelan que existen diferencias significativas entre el nivel de compromiso del grupo de hombres y del grupo de mujeres tanto si se evalúa antes de la simulación $\left(\mathrm{t}(101)=2.16, \mathrm{p}<.05 ; \mathrm{M}_{\text {Hом- }}\right.$ ${ }_{\text {BRES }}=7.50$, d.t. $=1.59 ; \mathrm{M}_{\text {MUJERES }}=6.75$, d.t. $\left.=1.22\right)$, como tras la misma $\left(\mathrm{t}(101)=2.29, \mathrm{p}<.05 ; \mathrm{M}_{\text {HOMBRES }}=7.87\right.$, d.t. $=.83 ; \mathrm{M}_{\text {MUJERES }}=7.27$, d.t.=1.33), siendo, en ambos casos, los hombres quienes muestran un mayor compromiso con la tarea.

Reacciones afectivas. El modelo de medidas repetidas para el factor sexo sobre las reacciones afectivas evaluadas antes y después de la simulación no resulta significativo. Las pruebas $t$ para muestras relacionadas muestran, sin embargo, que los cambios entre la medida antes y tras la simulación son significativos para el grupo de mujeres $\left(\mathrm{t}(83)=4.50, \mathrm{p}<.001 ; \mathrm{M}_{\mathrm{ANTES}}=3.01\right.$, d.t.=.84; $\mathrm{M}_{\text {DESPUÉS }}=2.47$, d.t.=1.02), mejorando su estado afectivo tras realizar la simulación. En el caso de los varones, las diferencias no resultan significativas. Las pruebas $t$ para muestras independientes tampoco revelan diferencias significativas entre las reacciones afectivas de hombres y mujeres, ni antes de la simulación ni tras la misma.

\section{Efecto del sexo sobre el desempeño en la simulación}

Por último, estamos interesados en explorar si existen diferencias significativas en el número de adoptadores logrados en la tarea entre hombres y mujeres. De nuevo llevamos a cabo un análisis de varianza (ANOVA) que revela que, aunque las varianzas de ambos grupos no son iguales, las diferencias son significativas $\left(F(1,101)=2.67, p<.10, \eta^{2}=.026\right)$. Así, el grupo de hombres alcanza un mejor desempeño final en la simulación $\left(\mathrm{M}_{\text {HOMBRES }}=7.44\right.$, d.t.=5.37) que el grupo de mujeres $\left(\mathrm{M}_{\mathrm{MUJERES}}=5.75\right.$, d.t.=3.45).

\section{Efecto del sexo, la autorregulación y la orientación hacia los errores sobre el desempeño}

Realizamos un path analysis para determinar el papel de la variable sexo y la orientación hacia los errores sobre el desempeño logrado en la tarea de simulación. En la Figura 2 se puede observar cómo el sexo ejerce un efecto directo sobre la autoeficacia para desempeñar tareas complejas. Así mismo, este mecanismo autorregulador junto a la dimensión de aprendizaje de la orientación hacia los errores determina el estado afectivo con el que el participante realiza la tarea. Estas tres variables (autoeficacia, aprendizaje de los errores y estado afectivo) determinan el desempeño final logrado $\left(\mathrm{R}^{2}=.273, \mathrm{~F}(4,90)=9.83, \mathrm{p}<.001\right)$. Los 
índices de ajuste del modelo resultaron ser adecuados $\left(\chi^{2}(4, \mathrm{~N}=104)=2.02, \mathrm{p} .=.73 ; \mathrm{NFI}=.97, \mathrm{CFI}=1.00\right.$, RMSEA =.01, $\mathrm{LO}=.01, \mathrm{HI}=.11$ ). En la figura se observa que aquellas personas con un juicio elevado de autoeficacia y mayor orientación a aprender de los errores presentan un estado afectivo menos negativo ( $\mathrm{B}=-.34$ y $\mathrm{B}=-30$; respectivamente). Así mismo, en la figura se percibe cómo logran un mejor desempeño quienes presentan un juicio más elevado de autoeficacia $(B=.25)$, tienen un estado afectivo menos negativo $(\mathrm{B}=-.40)$ y tienen una menor orientación a aprender de los errores $(\mathrm{B}=-.25)$. La orientación de los errores tendría un efecto positivo sobre el desempeño si hubiéramos evaluado la ejecución de la tarea durante un periodo de tiempo más amplio. Una alta orientación hacia los errores permitiría aprender de la experimentación en una tarea compleja de estas características durante un periodo de tiempo más amplio. La evaluación del impacto de la orientación de las metas hacia el aprendizaje junto a la orientación hacia los errores en tareas complejas requiere un mayor tiempo para aprender y obtener mejores resultados. Por el contrario, una orientación hacia el desempeño junto a una mayor evitación de la posibilidad de cometer errores conllevaría mejores resultados ante tareas complejas evaluadas durante un corto periodo de tiempo. En una circunstancia semejante, los individuos centrarían sus esfuerzos en obtener buenos resultados y evitar cometer errores.

\section{Discusión}

Los resultados de este estudio muestran que, a pesar de que los participantes manifiestan una orientación hacia el aprendizaje significativamente alta frente a una orientación hacia el desempeño y a mostrar competencia frente a los demás y de que ambas no correlacionan, la variable sexo aparece correlacionada únicamente con una tendencia hacia los resultados, siendo los varones quienes parecen estar más dirigidos a probar su propia capacidad, lo que estaría en línea con la investigación revisada (p. ej., Dweck y Leggett, 1988). Existe evidencia que sugiere que sugiere que los hom- bres valoran la competencia y la capacidad en mayor grado que las mujeres y que éstas valoran más el esfuerzo y la motivación (p. ej., Cooper, Burger y Good, 1981; McCrea, Hirt, Steele, Koch y West, 2000).

Otro aspecto a destacar son tanto las relaciones como el efecto de la variable sexo sobre los distintos mecanismos autorreguladores analizados. Así, son las chicas quiénes exhiben menores juicios de eficacia para la tarea y se proponen metas más bajas, tanto antes como después de realizarla, frente al grupo de los chicos. No obstante, son éstas quiénes manifiestan una actitud más positiva hacia comunicar los errores, a pesar de que se sienten menos autoeficaces a la hora de afrontar las dificultades en su trabajo cotidiano.

En este sentido, Kanfer y Ackerman (2000) abogaron por la necesidad de investigar cómo las variables demográficas influyen en los procesos motivacionales y los resultados laborales y por la influencia de la edad en la motivación laboral (Kanfer y Ackerman, 2004). Payne, Youngcourt y Beaubien (2007) también han evaluado las relaciones entre la orientación de las metas con la edad y el sexo, sin embargo, no encontraron relaciones bivariadas importantes. Podría ser interesante explorar en nuevos estudios la relación entre las dimensiones de la orientación de las metas y el sexo y la edad, dado que se han propuesto como moderadores de la relación orientación de las metas-desempeño (Midgley, Kaplan y Middleton, 2001) en tareas donde el feedback de fracaso y los errores sean comunes. Asimismo, resulta relevante la relación encontrada entre el sexo y la tendencia a comunicar los errores, dado que, hasta el momento no conocemos estudios en los que se haya explorado las diferencias en orientación hacia los errores de hombres y mujeres.

En este sentido, sería de interés tratar de identificar cómo se manejan estas cuestiones en el mundo laboral, dada la masiva incorporación de la mujer al trabajo remunerado y cada vez más en puestos de responsabilidad. Dado que entre las habilidades directivas para afrontar los nuevos retos empresariales destacan la capacidad de liderazgo, el trabajo en equipo, la creatividad, la toma de decisiones, la gestión del cambio, las habilidades de negociación, el autoaprendizaje y des-

Figura 2. Path analysis para explicar el desempeño alcanzado en una toma de decisiones compleja desde los mecanismos autorreguladores, la orientación a aprender de los errores y el sexo de los participantes (los valores indican los coeficientes beta significativos)

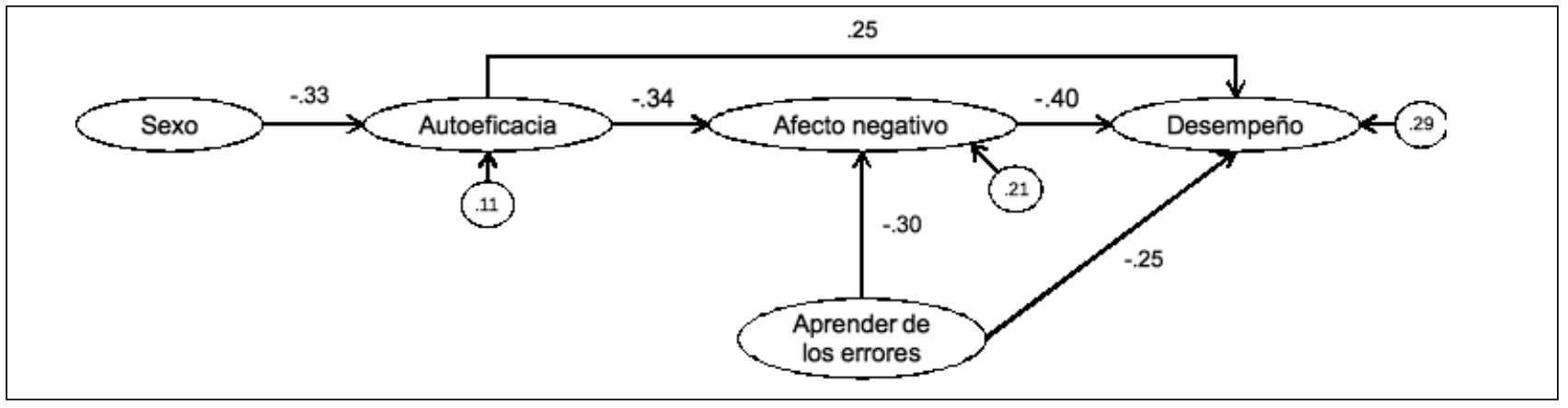


arrollo personal, y el dominio de las técnicas de comunicación, habilidades en muchos casos más representadas en mujeres que en hombres, lo que se ha denominado 'liderazgo transformacional'. En un entorno en constante cambio donde se valora la innovación y el riesgo es un valor, es preciso tener presente que en las organizaciones laborales de muchos países, incluido España, sigue predominando una cultura masculina. Los modelos y los criterios que sirven para medir la competencia y los requisitos para ocupar puestos de mando siguen estando claramente vinculados al estereotipo de varón (autoridad, decisión, independencia de criterio, fortaleza, preferencia por el riesgo).

Cuando las mujeres acceden a puestos intermedios y altos en las empresas adoptan, en muchos casos, algunas de las prácticas tradicionalmente masculinas. Esta desigualdad en las exigencias conlleva un proceso de exclusión por el que se dificulta, si no se impide, a las mujeres el acceso a recursos y posiciones valiosas dentro de la organización. Esta exclusión da lugar, a su vez, a que el sistema de géneros aún imperante en nuestras sociedades continúe imponiéndose en el ámbito laboral. El comportamiento de las mujeres sigue, entonces, siendo valorado en función de los estereotipos de género vigentes, a los que subyace la ya mencionada asociación entre poder y masculinidad. Si la sociedad avanza hacia una población mucho más educada, mucho más formada, con unas expectativas mucho más exigentes, la empresa no puede seguir alimentando los estereotipos de género, tampoco en el caso concreto de la toma de decisiones o la concepción del riesgo de hombres y mujeres.

\section{Extended Summary}

From an early age, children learn and incorporate beliefs associated with gender differentials which suggest that boys tend to be competitive, adventurous, enjoy tasks that involve risks, and are more interested in tasks which are related to mathematics, science and technology. At the same time, girls are considered cooperative, loving, charitable, intuitive, more emotional, less rational and more interested in tasks related to reading, writing, art and relationships. These stereotypical beliefs about gender are developed at a very early age (Burke, 1996), increase in complexity in childhood and adolescence and become more pronounced with age (Valiante, 2000). These stereotypes are sharing in many societies. In this context, the meta-analysis conducted by Byrnes, Miller and Schafer (1999) supports the idea that men are more likely to take risks than women. Gender differences vary according to context and age level, however, so men tend to encounter failure or other negative consequences more often than women, whereas women tend to experience success less often than they should.

On the other hand, there is a large body of research that examines the motivational patterns which unfold in both genders in achievement situations (e.g. Middleton \& Midgley, 1997; Pajares \& Schunk, 2001). Dweck $(1989,1999)$ provides studies showing that girls achieve the same level of performance as boys in mathematics during the early school years (and even outdo them in verbal tasks). During the last years of school and secondary education, however, boys outperform girls and stay ahead in maths.

Specifically, the fact that boys and girls often deploy different motivational patterns in maths and verbal comprehension, quite apart from the different skills they require, suggests that perhaps motivational patterns contribute to these discrepancies in achievement. In general, girls display less confident expectations, usually attribute failure to a lack of ability, show less preference for new and challenging tasks and face worse difficulties or confusion (Dweck, 1999).

The present study attempts to explore the role that gender plays in performance in complex tasks as they interact with dispositional variables such as the goal orientation directly involved in performance in achievement tasks, and error orientation. We also believe that the gender variable influences the self-regulatory mechanisms in place to explain performance in complex tasks more adequately (judgments of selfefficacy for the task, goals, emotional reactions and commitment to the task).

Goal orientation is a set of higher-order goals that help determine attitudes and behaviour in achievement situations (e.g. Dweck \& Leggett, 1988) and empirical studies have connected these goals with important outcome variables such as performance, seeking feedback and satisfaction (e.g. Elliot, McGregor, \& Gable, 1999; Vandewalle \& Cummings, 1997). Individuals with a higher level in terms of their learning goals guide their behaviour or dominance with the desire to develop their competence in the work they are doing, namely, to learn how to improve their capabilities. Simultaneously, those same people may differ in their level of goal orientation toward the performance in which they wish to demonstrate competence or avoid demonstrating their incompetence to others.

Moreover, key regulators of intentional behaviour or self-efficacy include the ability to perform the task, assessing performance against a target or standard and emotional reactions, including satisfaction. Self-efficacy not only ensures a level of effort and involvement in performing complex tasks, but is also the motivation to persist, despite failures, in addressing the risks and potential errors in the decision-making process. Those who maintain a high level of self-efficacy more easily develop an upward spiral in this process (Lindsey, Brass, \& Thomas, 1995). These spirals derive from their perceived ability to meet challenges, and their 
self-efficacy determines the level of effort and interest in achieving a higher goal level for best performance. In the light of the research reviewed, we suggest that the provisions relating to personal motivation for learning complex tasks such as error orientation, goal orientation and previous self-efficacy judgments are related to gender as well as self-regulatory mechanisms and performance in complex decision-making.

\section{Method}

\section{Sample}

The sample comprised a total of 104 students from different degree courses in psychology at the University of Salamanca, $46.2 \%$ in their first year and more than $80 \%$ being women. Some $96.2 \%$ of participants were under 26 years old.

\section{Task and Procedure}

The study was presented as a two-hour session of training and learning in decision-making by means of a simulator. Participation was rewarded financially. First, participants, who had been assured of strict anonymity, answered a questionnaire that included measures of some of the factors potentially determining performance with complex decision-making. Once everyone had completed the booklet, the task at hand was explained. It consisted of a simulation program, designed as a tool for training in decision-making, in which participants had to introduce an innovative element to the simulated organization to achieve the greatest possible number of adopters (Angehrn, 2004). The software was explained through a DEMO version presented in PowerPoint. Then participants answered a series of questions related to their perceived ability to achieve certain results in the task, its goals, their commitment and their emotional state when dealing with it. Finally, before leaving the session with the simulator they were asked to respond again to the questions referring to the self-regulatory mechanisms.

\section{Measures}

Dispositional variables: error orientation. We used a reduced version of the Error Orientation Questionnaire (EOQ) devised by Rybowiak, Frese, Garst, and Batinic (1999). Self-efficacy. We used an adaptation of microanalysis which has traditionally been used to assess the ability judgments in decisionmaking (Tabernero \& Wood, 1999; Wood \& Bandura, 1989). Goal Orientation. In this particular context we used a questionnaire adapted from Vandewalle's (1997) Work Domain Goal Orientation Instrument.
Self-regulatory mechanisms (measured before and after simulation): self-efficacy. It was assessed by six items that referred to beliefs about one's ability to reach different results in the simulation program. Selfset goals for the task. These were measured through two questions: one referring to the number of adopters recruited in the simulation time, and one to how many points they pursue. Goal Commitment. It was assessed through the items 'How committed do you feel to the goal selected' and 'How committed would you feel to a goal selected in the future?'. Affective Reactions. These were assessed through a semantic differential (Snider \& Osgood, 1969).

Performance in the simulation program. The program allowed us to collect data that can be considered as dependent variables in the study. In this paper we treated the number of adopters achieved as an indicator of performance.

\section{Results and Discussion}

The results of this study show that although the participants expressed a learning goal orientation significantly higher than performance goal orientation in competition with others and the two do not correlate, the variable gender was correlated only with a trend toward results (performance goal orientation) in men, who seem more geared towards testing their capacity, which would be in line with the research reviewed (e.g. Dweck \& Leggett, 1988). Evidence suggests that men value competence and ability more than women and that they appreciate effort and motivation more (e.g. Cooper \& Burger, 1981; McCrea, Hirt, Steele, Koch, $\&$ West, 2000).

Results showed that men exhibited lower self-efficacy in the task and set goals lower, both before and after the performance, than the group of women. Women, however, showed a more positive attitude in terms of communicating errors, although they were less self-efficacious when facing difficulties in their daily work.

In this context, Kanfer and Ackerman (2000) have advocated the need to investigate how demographic variables influence motivational processes and work performance and the influence of age on work motivation (Kanfer \& Ackerman, 2004). Payne, Youngcourt and Beaubien (2007) have also evaluated the relationship between goal orientation, age and gender; however, they found no significant bivariate relationships. It would be interesting to explore in further studies the relationship between the dimensions of goal orientation, gender and age as moderators of the relationship between goal orientation and performance (Midgley, Kaplan, \& Middleton, 2001) in tasks where feedback from failure and mistakes are common. It is also important to explore the relationship found between gender and tendency to report errors, since to date 
there are no known studies that have explored the differences in error orientation of men and women.

In this context, it would be interesting to try to identify how to handle these issues in the workplace, given the massive recruitment of women to paid work and increasingly responsible positions. Among the managerial skills required to meet new business challenges are leadership skills, teamwork, creativity, decisionmaking, change management, negotiation skills, independent learning, personal development and mastery of communication techniques, skills in many cases more representative of women than men, a phenomenon which has been termed 'transformational leadership'. In a constantly changing environment that values ??innovation and risk, it should be noted that labour organizations in many countries, including Spain, retain a dominant male culture. The models and the criteria used to measure the skills and requirements for positions of command are still strongly linked to the stereotype of the male (authority, determination, independence of mind, strength, predisposition to risk adoption).

When women have access to intermediate and senior positions in companies they adopt, in many cases, some of the traditionally male practices. This unevenness involves a process of exclusion that hinders, or even prevents, women's access to resources and valuable positions within the organization. This exclusion leads, in turn, to the gender system prevailing in our societies continuing to be imposed in the workplace. The behaviour of women is therefore assessed in terms of existing gender stereotypes which underlie the aforementioned association between power and masculinity. If society is moving towards a much more educated population whose expectations are far more demanding, companies cannot continue to feed gender stereotypes.

\section{Referencias}

Angehrn, A. (2004). The EIS Simulation: The Change, IT Innovation and People Management Challenge (versión 2.5) [Software de ordenador]. Fontainebleau, Francia: AlphaLabs.

Bandura, A. (1986). Social foundations of thought and action: A social cognitive theory. Englewood Cliffs, NJ.: Prentice-Hall.

Bandura, A. (1997). Self-efficacy: The exercise of control. New York: Freeman and Company.

Bandura, A. (1999). Autoeficacia. Cómo afrontamos los cambios de la sociedad actual. Bilbao: Desclée De Brouwer.

Burke, P. (1996). Gender shock: Exploding the myths of male and female. Nueva York: Anchor Books.

Byrnes, J. P. (1998). The nature and development of decision-making: A self-regulation model. Hillsdale, NJ: Erlbaum.
Byrnes, J. P., Miller, D. C. y Schafer, W. D. (1999). Gender differences in risk taking: A meta-analysis. Psychological Bulletin, 125, 367-383.

Cooper, H. M., Burger, J. M. y Good, T. L. (1981). Gender differences in the academic locus of control beliefs of young children. Journal of Personality and Social Psychology, 40, 562-572.

Debowski, S., Wood, R. E. y Bandura, A. (2001). Impact of guided exploration and enactive exploration on self-regulatory mechanisms and information acquisition through electronic search. Journal of Applied Psychology, 86, 1129-1141.

Dweck, C. S. (1989). Motivation. En A. Lesgold y R. Glaser (Eds.), Foundations for a Psychology of Education. Hillsdale, NJ: Lawrence Erlbaum and Associates.

Dweck, C. S. y Leggett, E. L. (1988). A social-cognitive approach to motivation and personality. Psychological Review, 95, 256-273.

Dweck, C. S.(1999). Self-theories. Their role in motivation, personality and development. Ann Arbor, MI: Taylor \& Francis.

Elliot, A. J., McGregor, H. A. y Gable, S. (1999). Achievement goals, study strategies and exam performance. Journal of Educational Psychology, 91, 549-563.

Elliot, E. S. y Dweck, C. S. (1988). Goals: An approach to motivation and achievement. Journal of Personality and Social Psychology, 54, 5-12.

Hackett, G. y Betz, N. E. (1991). Self-efficacy expectations in the career choices of college students. For D. Schunk y J. Meese (Eds.), Student perceptions in the classroom: Causes and consequences. Hillsdale, NJ: Erlbaum (pp. 229-246).

Irwin, C. E. y Millstein, S. G. (1991). Correlates and predictors of risk-taking behavior. En L. P. Lipsitt y L. L. Mitnick (Eds.), Self-regulatory behavior and risk-taking: Causes and consequences (pp. 3-21). Norwood, NJ: Ablex.

Kanfer, R. y Ackerman, P. L. (2000). Individual differences in work motivation: Further explorations of a trait framework. Applied Psychology: An International Review, 49, 470-482.

Kanfer, R. y Ackerman, P. L. (2004). Aging, adult development, and work motivation. Academy of Management Review, 29, 440-458.

Kelling, G. W., Zirkes, R. y Myerowitz, D. (1976). Risk as value: A switch of set hypothesis. Psychological Reports, $38,655-658$.

Lindsley, D. H., Brass, D. J. y Thomas, J. B. (1995). Efficacy-performance spirals: A multi-level perspective. Academy of Management Review, 20, 645-678.

McCrea, S. M., Hirt, E. R., Steele, N. L., Koch, K. A. y West, S. T. (2000). Sex differences in beliefs about effort versus competence. Manuscrito no publicado, Indiana University Bloomington.

Middleton, M. J. y Migdley, C. (1997). Avoiding the domostration of lack of ability: An underexplored aspect of goal theory. Journal of Educational Psychology, 89, 710-718

Midgley, C., Kaplan, A., y Middleton, M. J. (2001). 
Performance-approach goals: Good for what, for whom, under what circumstances, and at what cost? Journal of Educational Psychology, 93, 77-86.

Pajares, F. y Schunk, D. H. (2001). Self-beliefs and school success: Self-efficacy, self-concept, and school achievement. En R. Riding y S. Rayner (Eds.), Perception (pp. 239-266). London: Ablex Publishing.

Payne, S. C., Youngcourt, S. S. y Beaubien, J. M. (2007). A meta-analytic examination of the goal orientation nomological net. Journal of Applied Psychology, 92, 128-150.

Rybowiak, V., Garst, H., Frese, M. y Batinic, B. (1999). Error orientation questionnaire (EOQ): Reliability, validity, and different language equivalence. Journal of Organizational Behavior, 20, 527-547.

Sarrió, M., Barberá, E., Ramos, A. y Candela, C. (2002). El techo de cristal en la promoción profesional de las mujeres. Revista de Psicología Social, 17, 167-182.

Snider, J. G. y Osgood, C. E. (1969). Semantic differential technique: A sourcebook. Chicago: Aldine Publishing Company.

Tabernero, C. y Wood, R. E. (1999). Implicit theories versus the social construal of ability in self-regulation and performance on a complex task. Organizational Behavior and Human Decision Processes, 78, 104-127.

Valiante, G. (2000). Writing Self-efficacy and Gender Orientation. A developmental Perspective. A Dissertation Proposal. Atlanta: Emory University.

VandeWalle, D. (1997). Development and validation of a work domain goal orientation instrument. Educational and Psychological Measurement, 57, 995-1015.

VandeWalle, D. y Cummings, L. L. (1997). A test of the influence of goal orientation on the feedback-seeking process. Journal of Applied Psychology, 82, 390-400.

Virtanen, P. y Nevgi, A. (2010). Disciplinary and gender differences among higher education students in self-regulated learning strategies. Educational Psychology: An International Journal of Experimental Educational Psychology, 30, 323-347.

Weller, J. A., Levin, I. P. y Denburg, N. L. (2011). Trajectory of risky decision making for potential gains and losses from ages 5 to 85. Journal of Behavioral Decision Making, 24.

Weller; J. A., Levin, I. P. y Bechara, A. (2009). Do individual differences in Iowa Gambling Task performance predict adaptive decision making for risky gains and losses? Journal of Clinical and Experimental Neuropsychology, 32, 141-150.

Wood, R. E. y Bandura, A. (1989). Impact of conceptions of ability on self-regulatory mechanisms and complex decision making. Journal of Personality and Social Psychology, 56, 407-415.

Wood, R. E., Kakebeeke, B., Debowski, S. y Frese, M. (2000). The impact of enactive exploration on intrinsic motivation, strategy, and performance in electronic search. Applied Psychology: An International Review, 49, 263-283.

Manuscrito Recibido: 15/12/2010

Revisión Recibida: 22/03/2011

Aceptado: 22/03/2011 